\title{
Magnetic field concentration with coaxial silicon nanocylinders in the optical spectral range
}

\author{
Ksenila V. Baryshnikova, , ${ }^{1, *}$ Andrey Novitsky, ${ }^{2,3}$ Andrey B. Evlyukhin, ${ }^{1,4}$ and Alexander S. Shalin ${ }^{1,5,6}$ \\ 'Laboratory "Nano-optomechanics," ITMO University, 49 Kronverksky Pr., 197101 St. Petersburg, Russia \\ ${ }^{2}$ DTU Fotonik, Technical University of Denmark, Orsteds Plads 343, DK-2800 Kongens Lyngby, Denmark \\ ${ }^{3}$ Department of Theoretical Physics and Astrophysics, Belarusian State University, Nezavisimosti Ave. 4, 220030 Minsk, Belarus \\ ${ }^{4}$ Laser Zentrum Hannover e.V., Hollerithallee 8, D-30419 Hannover, Germany \\ ${ }^{5}$ Kotel'nikov Institute of Radio Engineering and Electronics of Russian Academy of Sciences (Ulyanovsk branch), \\ Goncharova str. 48/2, 432071 Ulyanovsk, Russia \\ ${ }^{6}$ Ulyanovsk State University, Lev Tolstoy str. 42, 432017 Ulyanovsk, Russia \\ *Corresponding author: k.baryshnikova@optomech.ifmo.ru
}

Received 14 February 2017; revised 31 March 2017; accepted 7 April 2017; posted 11 April 2017 (Doc. ID 286612); published 1 May 2017

\begin{abstract}
Resonant magnetic energy accumulation is theoretically investigated in the optical and near-infrared regions. It is demonstrated that the silicon nanocylinders with and without coaxial through holes can be used for the control and manipulation of optical magnetic fields, providing up to 26-fold enhancement of these fields for the considered system. Magnetic field distributions and dependence on the parameters of nanocylinders are revealed at the wavelengths of magnetic dipole and quadrupole resonances responsible for the enhancement. The obtained results can be applied, for example, to designing nanoantennas for the detection of atoms with magnetic optical transitions. (๑) 2017 Optical Society of America
\end{abstract}

OCIS codes: (260.5740) Resonance; (290.4020) Mie theory; (350.4238) Nanophotonics and photonic crystals; (290.0290) Scattering.

\section{INTRODUCTION}

The concentration of light in small volumes is a topic of extensive investigation, suggesting a broad range of possible applications, like SERS, imaging and visualization, photovoltaic and photonics applications, and so on [1-4]. While the performances of conventional optical elements cannot lead to substantial field concentrations below the classical diffraction limit, various auxiliary nanostructures, operating within the near field, could deliver deep subwavelength enhancement. In particular, negative permittivity nanoparticles, supporting localized plasmon resonances, are capable of concentrating electric fields at nanoscale volumes [5]. Electromagnetic energy in this case is stored in the electrical field during one-half of an oscillation period, while electron displacement in negative epsilon materials accumulates the energy during its second half. Plasmonic particles have an electric dipole response and concentrate fields at their close vicinity and, as a result, are widely employed for enhancing fluorescence [6], improving sensing [7], achieving nanoscale lasing [8], nanopositioning with optical tweezing [9-11], and for many other applications. An approach for manipulating the magnetic field component of light relies on exciting magnetic Mie resonances in high-refractive-index dielectric particles [12-15]. In this case, circular displacement currents in the fully retarded regime create effective magnetic dipolar and higher-order resonances [16,17]. Magnetic resonances of this kind were studied in various configurations, aiming to tailor optical properties of micro- and nanostructures by exploring and adjusting a set of their geometrical parameters. While spherical geometries have only 2 degrees of freedom, their radius and material parameters (silicon and germanium are the most frequently used), cylinders provide a higher degree of flexibility in optical response tuning. The phenomenon in high-index particles relies on retardation effects, and, as a result, fields are attracted to regions containing higher permittivity materials, similar to the waveguiding phenomenon [18]. Field concentrations in those geometries are achieved inside high-refractiveindex materials and, as a result, are inaccessible by external probes. However, electromagnetic fields could be accessible in small gaps inside high-refractive-index particles [19]. Note that the magnetic responses of particles with hollows had been considered previously [14,20-23], but only with the view of the scattering problem, while magnetic field concentrations had not been investigated. In this paper, we investigate the magnetic field distribution inside silicon nanocylinders with and without coaxial through holes to obtain high-efficient magnetic hot-spots (MHSs). 
The paper is organized as follows. In Section 2, we carry out the optimization of the geometrical parameters of nanocylinders, aiming to achieve high magnetic field concentrations. In Section 3, the geometry of the silicon nanocylinders is changed to include the coaxial air void where the high magnetic field concentration is to be achieved. All numerical calculations are done with the help of the finite element method, realized in Comsol Multiphysics (available at https://www.comsol.com). A multipole analysis is performed with the help of the methods suggested in [24]. The data of the crystalline silicon dielectric function are taken from [25].

\section{MAGNETIC HOT-SPOTS IN SILICON NANOCYLINDERS}

We consider a silicon cylinder excitation by a plane wave of linear polarization and two different propagation directions: along (frontal excitation) and perpendicular (lateral excitation) to the cylinder's axis (see Fig. 1). Another case, when the wave electric field in Fig. 1(b) oscillates along cylinder's axis, results in magnetic hot-spots very similar to those excited in frontal configuration, which, therefore, are not discussed here.

In our numerical simulations, in order to realize magnetic hot-spots inside the cylinders in the visible and near-infrared ranges, we varied the height and radius of the cylindrical nanoparticles in the ranges from $80-250 \mathrm{~nm}$ and $30-190 \mathrm{~nm}$, respectively [26]. The obtained results can be approximated to a wider range of geometrical parameters. We investigate the spectral dependencies of the magnetic field maximum located on the cylinder's axis, and it is shown that these dependencies can have several maxima depending on the cylinder's geometry (see details in the next section). So, let us first consider the first resonance, i.e., the resonance corresponding to the longest wavelength. It is worth noting that resonant MHSs can correlate to the different multipole resonances, which will be considered below in this paper.

The light wavelength of the first resonance and corresponding magnetic field maximum, normalized to the amplitude of the incident magnetic field, are shown in Fig. 2 as a function of the cylinder's height and radius. It can be seen that the resonant wavelength and the value of magnetic field on the first resonance are dependent on the irradiation conditions.

Multipole analysis can help to interpret the peaks of the local fields in the nanoparticle and follow the evolution of the resonances by changing the structure's geometrical parameters (see next sections). The method of scattering cross-section multipole

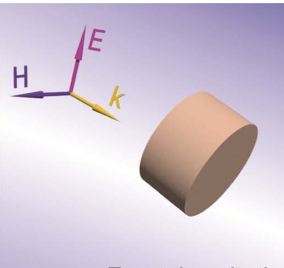

Frontal excitation
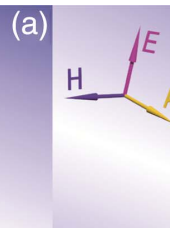

Lateral excitation
Fig. 1. Cylinders, irradiated by a plane wave, which propagates (a) along the cylinder axis (frontal excitation) and (b) perpendicular to the cylinder axis (lateral excitation).

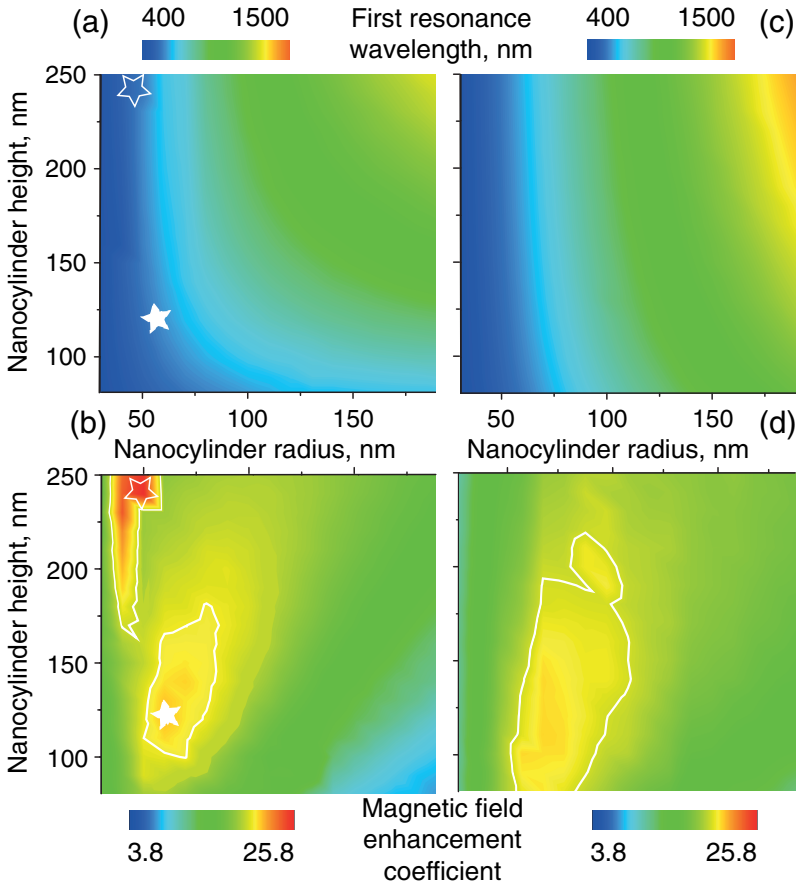

Fig. 2. (a) and (c) Maps of the light wavelength of the first resonance (i.e., the resonance corresponding to the longest wavelength) as a function of the cylinder size parameters: (a) for the frontal excitation and (c) for the lateral excitation. (b) and (d) Maps of magnetic field enhancement coefficient (normalized magnetic field maximum) at the first resonance as a function of the cylinder size parameters: (b) for the frontal excitation and (d) for the lateral excitation. The white stars (a) and (b) correspond to the parameters chosen for the MHS illustration in Fig. 3. The white contoured islands (b) and (d) highlight the areas where the enhancement coefficient is greater than 20 .

decomposition is proposed in [24], based on the integration of polarization density in the nanoparticle volume, which can be made by post-processing in the Comsol Multiphysics software. At first, our multipole analysis (see Fig. 3 for details) shows that the first resonance and the consequent magnetic field enhancement always correspond to the resonance of the magnetic dipole moment. In the case of a cylinder with $R=60 \mathrm{~nm}, H=$ $120 \mathrm{~nm}$, the maximum of the magnetic field inside the nanoparticles can exceed twenty times the magnetic field amplitude of the incident light [see white contour in Fig. 2(b)]. The distribution of the magnetic field enhancement coefficient in Fig. 2(d) is similar to the distribution shown in Fig. 2(b), but the white contours, highlighting areas where the magnetic field enhancement coefficient is greater than 20 , are different on these two maps. The asymmetrical shape of the contours is described by the dispersion of the silicon dielectric function. One of the contours on the map in Fig. 2(b) has no analogue to the map in Fig. 2(d). This area corresponds to the cylinders with big aspect ratios. In these particles, the magnetic quadrupole resonance is spectrally close to the magnetic dipole one (see Fig. 3), and their interplay can produce a highly effective MHS, where the value of the enhancement coefficient can be up to 26 times [see Fig. 2(b)]. Characteristic distributions of the magnetic field inside the nanoparticles for the two cases marked by the white stars in Figs. 2(a) and 2(b) are shown in Fig. 3. 


\section{MAGNETIC HOT-SPOTS IN COAXIAL SILICON NANOCYLINDERS WITH VOID CORE}

Above, we consider the MHSs inside the nanoparticle volume. Meanwhile, for practical applications, it is important to have direct access to the MHSs. Several approaches have been suggested for these purposes previously. For example, an MHS can be obtained in a small gap between high-index nanoparticles $[27,28]$ or in the through cavity $[14,23,29]$. While the magnetic field enhancement coefficient between the particles is always much smaller than inside a particle, the localization of the magnetic field in the particle's cavity can be almost as effective as in particle's volume.

\section{A. Magnetic Hot-Spots of the First Resonance}

In the case of high-refractive-index dielectric (silicon) nanocylinders, when a strong magnetic field concentrates inside a nanoparticle (Fig. 3), they could be accessed by using a coaxial through hole (or cavity): see Fig. 4(a) (frontal irradiation) and Fig. 4(b) (lateral irradiation). Such holes can be produced by focused ion-beam milling or electron beam lithography and reactive ion etching $[14,23]$. In Fig. 4, the results of the numerical simulations for cylinders with radius $R=60 \mathrm{~nm}$ and height $H=120 \mathrm{~nm}$ with through holes are presented for both types of irradiation. Note that these geometrical parameters correspond to the maximal magnetic field enhancement up to 22 times in Figs. 2(b) and 2(d), which is caused by magnetic dipole resonance. The dependencies similar to those in Fig. 4 take place for nanocylinders with other parameters if the magnetic dipole resonance is excited.

As is clearly seen from Fig. 4, the distributions of the magnetic field in particles with small cavities are nearly the same as

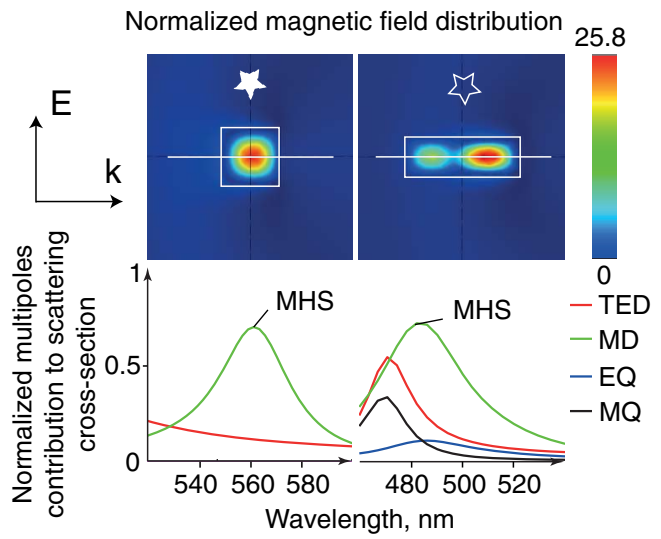

Fig. 3. Top: normalized magnetic field distribution for the first resonance in nanocylinders in the case of the frontal excitation (side view). The white lines indicate the cylinders' axes. All cases are shown in Figs. 2(a) and 2(b) by the white stars. Bottom: multipole decomposition of cylinders' scattering cross section in the vicinity of the resonant wavelength. Several multipoles with rather significant contributions are shown: total electric dipole (TED, red line), magnetic dipole ( $\mathrm{MD}$, green line), electric quadrupole (EQ, blue line), and magnetic quadrupole (MQ, black line). Wavelength of the first resonance of MHS on the cylinder axis is marked by "MHS." Parameters of the nanocylinders: left-radius $R=60 \mathrm{~nm}$, height $H=120 \mathrm{~nm}$, wavelength $564 \mathrm{~nm}$; right $-R=40 \mathrm{~nm}, H=230 \mathrm{~nm}$, wavelength $482 \mathrm{~nm}$.

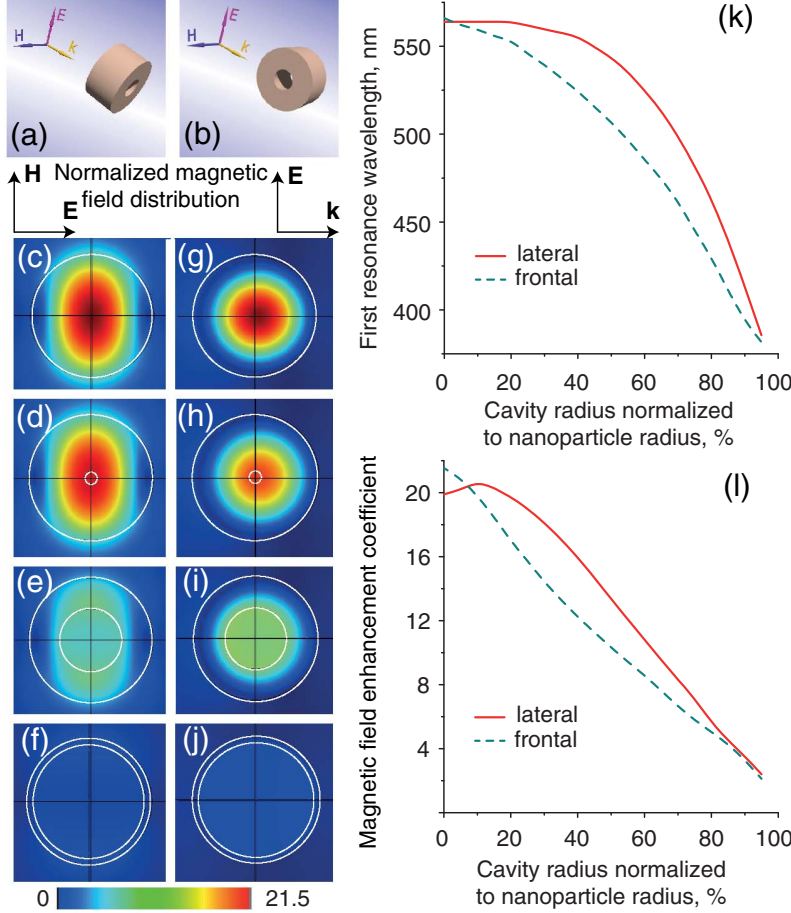

Fig. 4. Frontal (a) and lateral (b) excitations of a cylinder with a coaxial through hole. (c)-(j) Distributions of normalized magnetic field for the first resonance in the cylinders with radius $R=$ $60 \mathrm{~nm}$ and height $H=120 \mathrm{~nm}$ (these parameters correspond to the maximal magnetic field enhancement in Figs. 2(b) and 2(d) and cylinders with coaxial holes of different radii. (c)-(f) Frontal and $(\mathrm{g})-(\mathrm{j})$ lateral excitations. (d) Cavity radius is $6 \mathrm{~nm}$, resonant wavelength is $566 \mathrm{~nm}$; (e) cavity radius is $30 \mathrm{~nm}$, resonant wavelength is $507 \mathrm{~nm}$; (f) cavity radius is $54 \mathrm{~nm}$, resonant wavelength is $494 \mathrm{~nm}$; (h) cavity radius is $6 \mathrm{~nm}$, resonant wavelength is $564 \mathrm{~nm}$; (i) cavity radius is $30 \mathrm{~nm}$, resonant wavelength is $543 \mathrm{~nm}$; and (j) cavity radius is $54 \mathrm{~nm}$, resonant wavelength is $440 \mathrm{~nm}$. (k) The wavelengths corresponding to the first resonance as a function of the hole radius. (l) Magnetic field enhancement coefficient in the hole as a function of its radius.

for the nanoparticle without a cavity [Figs. 4(c)-4(d) and $4(\mathrm{~g})-4(\mathrm{~h})]$. The magnitude of the magnetic field is quite homogeneous on the frontal cross section and has a Lorentz-like maximum near the middle of the cylinder's axis. The magnetic field in the cavity becomes weaker with the increase of the cavity radius [see Figs. 4(d) $-4(\mathrm{f})$ and $4(\mathrm{~h})-4(\mathrm{j})$ ]. However, even for a silicon shell of $6 \mathrm{~nm}$ thickness (10\% of the outer cylinder's radius), the magnetic field magnitude in the cavity is enhanced up to 2 times for the both frontal and lateral irradiation conditions. When the radius of cavity is larger than $6 \mathrm{~nm}$, the lateral excitation of nanocylinders is more prospective for realizing strong magnetic fields [Fig. 4(l)]. It originates from the fact that the circular character of the displacement currents is not changed for the lateral irradiation. In the case of the frontal excitation, the circular currents are discontinuous, which leads to the more rapid destruction of the MHSs with the increase of the cavity radius. The wavelength corresponding to the MHS excitation decreases monotonically when the size of the cavity goes up in both cases [Fig. $4(\mathrm{k})$ ]. Importantly, the dependencies 
presented in Figs. 4(k) and 4(l) allow us to construct a system with the required levels of magnetic field enhancement in a through hole for a certain spectral range.

\section{B. Magnetic Hot-Spots of Higher-Order Resonances}

Now, let us consider the magnetic field enhancement at higherorder resonances with wavelengths lower than the wavelength of the first resonance. Let us choose, for example, a cylinder with $H=180 \mathrm{~nm}$ and $R=190 \mathrm{~nm}$, but the same dependencies can be obtained for other geometrical parameters. The spectral dependencies of the magnetic field enhancement coefficient on the axis of a cylinder with (black dotted line) and without (black solid line) a hole are presented in Fig. 5 for

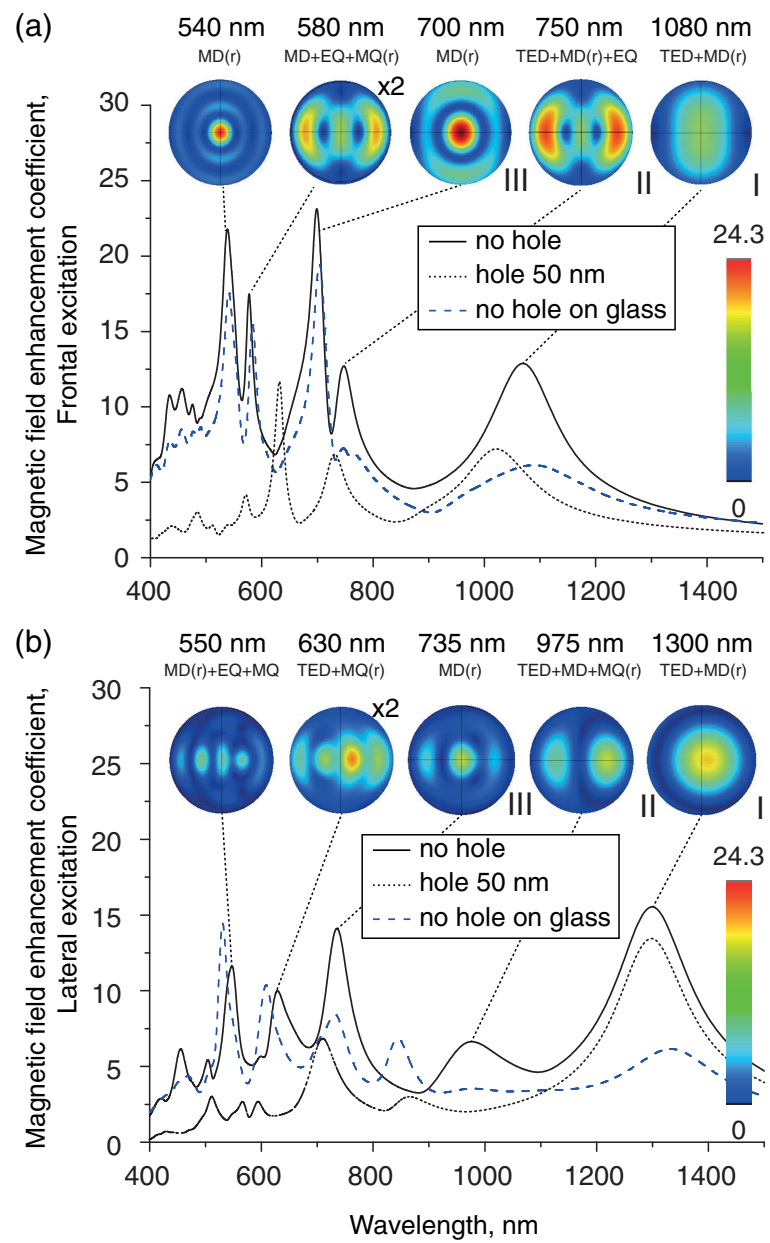

Fig. 5. Spectra of magnetic field enhancement coefficient for the cylinder without a hole in vacuum (black solid line), for the cylinder with a hole of radius $50 \mathrm{~nm}$ in vacuum (black dotted line), and for the cylinder without a hole on the glass substrate (blue dashed line) for frontal excitation (a) and for lateral excitation (b). Insets show distributions of normalized magnetic field at the resonance wavelengths at the plane crossing the MHS's center, where the magnetic field is maximal. Index " $\times 2$ " corresponds to the two maxima on the cylinder's axis. All results correspond to the cylinders with $R=190 \mathrm{~nm}$, $H=180 \mathrm{~nm}$, and without a hole. The symbols "I," "II," and "III" indicate resonances whose evolutions are observed in Fig. 7. The most significant multipoles contributions for each resonant wavelength are shown (see Fig. 6 for details). frontal (a) and lateral (b) excitation. The insets show the distributions of the normalized magnetic field, corresponding to the resonances of a particle without a hole at the plane crossing the MHS center, where the magnetic field is maximal. Also, Fig. 5 demonstrates the magnetic field enhancement coefficient in the particle without a hole located on a glass substrate (blue dashed line).

The volume, where the magnetic field is concentrated, decreases for high-order resonances compared with the first resonance, and the MHSs can split into two or more hot-spots along the cylinder's axis (for example, the " $\times 2$ " resonances in Fig. 5 correspond to the two similar hot-spots along the axis: the field distribution corresponding to one of the magnetic field maxima is shown) or perpendicular to the cylinder's axis. For the lateral excitation, the magnetic field enhancement is larger for the first resonance, whereas for the frontal excitation, the resonance at $630 \mathrm{~nm}$ provides stronger MHSs with a magnetic field enhancement coefficient up to 25 times. Similar dependencies are observed for the cylinders from the considered range of parameters; however, for small cylinders, high-order resonances can move into the shorter wavelength region or even

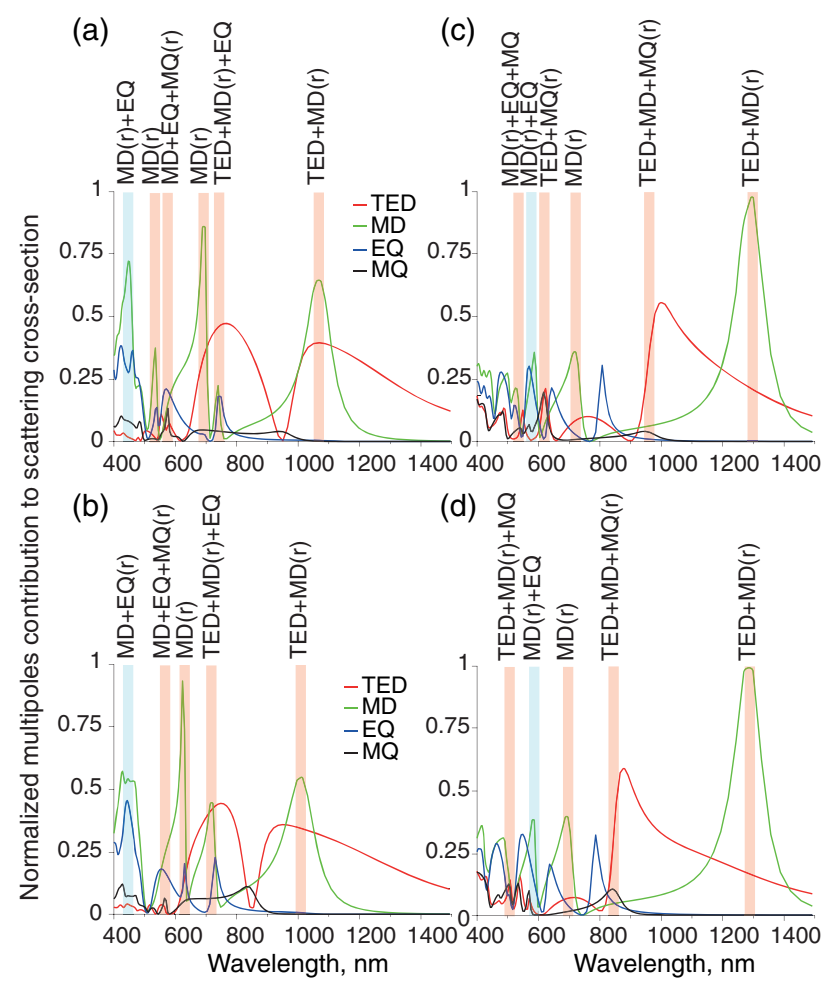

Fig. 6. Normalized multipole contributions to the scattering cross section of a cylinder with $H=180 \mathrm{~nm}, R=190 \mathrm{~nm}$ in free space with the following conditions: (a) particle with no hole, frontal irradiation; (b) particle with a hole of $50 \mathrm{~nm}$ radius, frontal irradiation; (c) particle with no hole, lateral irradiation; and (d) particle with a hole of $50 \mathrm{~nm}$ radius, frontal irradiation. Red vertical lines highlight spectral regions where MHSs take place. Blue lines highlight spectral regions where MHSs are suppressed while the magnetic dipole moment is resonantly increased. The most significant multipole contributions for each area are shown. The abbreviations on the legends are the same as in Fig. 3. The resonant contributions are marked by "(r)." 
disappear because of the strong changes of the silicon refractive index in the ultraviolet spectral range.

Higher-order resonances correspond to more inhomogeneous distributions of the displacement currents inside nanocylinders (see Fig. 5). Therefore, these resonances are very sensitive to a cylinder's defects, like a cavity or coaxial hole. The black dotted lines in Fig. 5 illustrate the magnetic field enhancement on the cylinder's axis in the case of a hole's presence (50 $\mathrm{nm}$ radius). Interestingly, some resonances stay quite strong, while others almost disappear when the hole increases. Different characters of dependencies can be explained with help of the multipole decomposition method.

An accurate analysis of the multipole decomposition of a cylinder's scattering cross section up to the magnetic quadrupole is done (see Fig. 6). It shows that the MHS resonant excitation can correlate with different multipole resonances, such as the magnetic dipole and magnetic quadrupole (red vertical lines in Fig. 6). It is interesting that the electric quadrupole enhancement simultaneously with the magnetic dipole one deeply suppresses the MHS excitation (blue vertical lines in Fig. 6). This result shows that not only do magnetic multipole resonances produce resonant MHSs, but the combination of multipoles is important. Some MHS excitation can be associated with higher-order multipoles not calculated here.

The dependencies of the resonant wavelengths and the magnetic field enhancement coefficients on three long-wavelength resonances is investigated. These resonances correspond to the wavelengths 1080, 750, and $700 \mathrm{~nm}$ for frontal irradiation and to the wavelengths 1300,975 , and $735 \mathrm{~nm}$ for lateral irradiation of a particle without a hole (see Fig. 5, resonances marked by the symbols "I," "II," and "III"). They are quite stable when the cavity increases, so their evolution is quite clear in contrast with lower-wavelength resonances. The magnetic field enhancement coefficient and resonant wavelength are being changed with the variation of the cavity size (Fig. 7). These dependencies could be useful for the construction of the magnetic field's control system.

Finally, in practical applications, the nanoparticles are usually placed on the substrate. When the refractive index and absorption coefficient of a substrate are small (as, for example, with a glass), its influence on the MHS properties is rather insignificant, and most of the resonant frequencies stay the same (blue dashed line in Fig. 5) [28].

In conclusion, we have investigated the magnetic field distribution inside silicon nanocylinders with and without coaxial through holes using full-wave numerical simulations. Two cases of irradiation have been included: (1) along and (2) perpendicular to the cylinder's axis. It has been found that in both cases, highly efficient MHSs corresponding to different cylinder multipole moments could be excited. The conditions for obtaining MHSs have been investigated in detail. Multipole decomposition of scattering cross-section spectra has been exploited to interpret the peculiarities of MHS excitation. Being quite tunable, the system allows us to control and manipulate the magnitudes of magnetic field enhancement and resonant wavelengths in broad spectral ranges. These results could be useful for different scientific areas, such as nanophotonics and nano-optomechanics, where MHSs can be used for controlling,
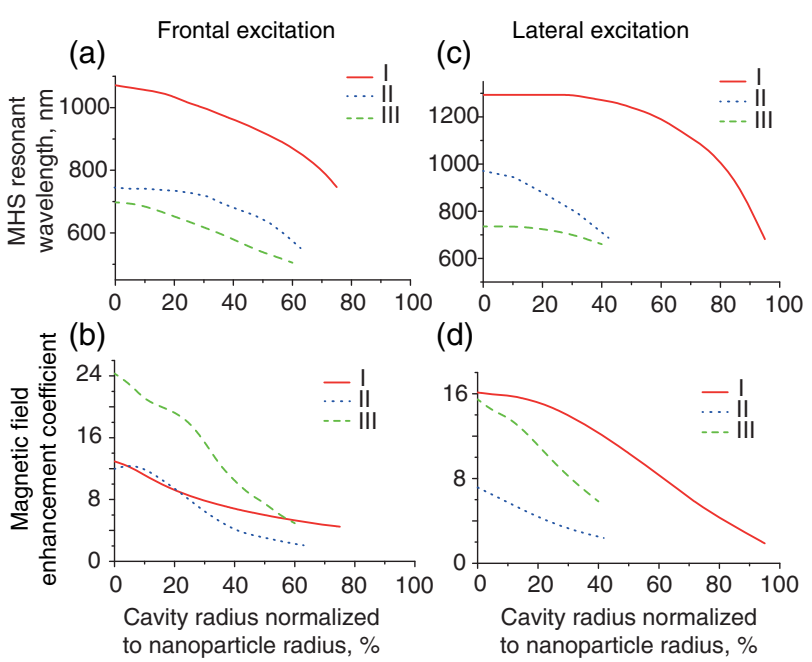

(d)

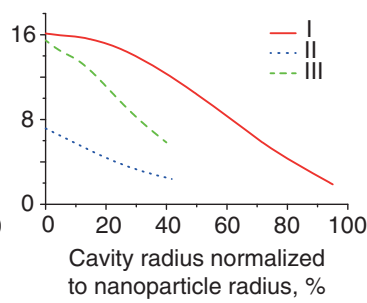

Fig. 7. (a) and (b) Dependencies of the resonant wavelengths and magnetic field enhancement coefficients on the hole radius for the resonances marked by "I," "II," and "III" in Fig. 5 for the case of frontal excitation. (c) and (d) The same, but for lateral excitation. All the results are obtained for a cylinder with $R=190 \mathrm{~nm}, H=180 \mathrm{~nm}$ in free space.

trapping, and detecting high-refractive-index dielectric nanoparticles and molecules with magnetic transitions.

Funding. Russian Foundation for Basic Research (RFBR) (16-52-00112); Russian Science Foundation (RSF) (16-1210287); Deutsche Forschungsgemeinschaft (DFG) (EV 220/2-1).

Acknowledgment. The calculations of multipole moments have been supported by the Russian Science Foundation Grant No. 16-12-10287. A. S. acknowledges the support of the President of the Russian Federation in the frame of Scholarship SP-4248.2016.1 and the support of the Ministry of Education and Science of the Russian Federation. K. B.'s research was partially supported by FASIE. A. N. acknowledges the financial support from the Belarusian Republican Foundation for Fundamental Research (Grant No. F16R-049) and the partial financial support from the Villum Fonden (DarkSILD project).

\section{REFERENCES}

1. D. Radziuk and H. Moehwald, "Prospects for plasmonic hot spots in single molecule SERS towards the chemical imaging of live cells," Phys. Chem. Chem. Phys. 17, 21072-21093 (2015).

2. A. Nazir, S. Panaro, R. Proietti Zaccaria, C. Liberale, F. De Angelis, and A. Toma, "Fano coil-type resonance for magnetic hot-spot generation," Nano Lett. 14, 3166-3171 (2014).

3. F. Pratesi, M. Burresi, F. Riboli, K. Vynck, and D. S. Wiersma, "Disordered photonic structures for light harvesting in solar cells," Opt. Express 21, A460-A468 (2013).

4. P. Ginzburg, D. Arbel, and M. Orenstein, "Gap plasmon polariton structure for very efficient microscale-to-nanoscale interfacing," Opt. Lett. 31, 3288-3290 (2006).

5. D. K. Gramotnev and S. I. Bozhevolnyi, "Plasmonics beyond the diffraction limit," Nat. Photonics 4, 83-91 (2010).

6. M. Bauch, K. Toma, M. Toma, Q. Zhang, and J. Dostalek, "Plasmonenhanced fluorescence biosensors: a review," Plasmonics 9 , 781-799 (2014). 
7. K. A. Willets and R. P. Van Duyne, "Localized surface plasmon resonance spectroscopy and sensing," Annu. Rev. Phys. Chem. 58, 267-297 (2007).

8. R. F. Oulton, V. J. Sorger, T. Zentgraf, R.-M. Ma, C. Gladden, L. Dai, G. Bartal, and X. Zhang, "Plasmon lasers at deep subwavelength scale," Nature 461, 629-632 (2009).

9. M. Righini, G. Volpe, C. Girard, D. Petrov, and R. Quidant, "Surface plasmon optical tweezers: Tunable optical manipulation in the femtonewton range," Phys. Rev. Lett. 100, 8-11 (2008).

10. A. A. Bogdanov, A. S. Shalin, and P. Ginzburg, "Optical forces in nanorod metamaterial," Sci. Rep. 5, 15846 (2015).

11. A. S. Shalin, S. V. Sukhov, A. A. Bogdanov, P. A. Belov, and P. Ginzburg, "Optical pulling forces in hyperbolic metamaterials," Phys. Rev. A 91, 1-6 (2015).

12. A. B. Evlyukhin, C. Reinhardt, A. Seidel, B. S. Luk'Yanchuk, and B. N. Chichkov, "Optical response features of Si-nanoparticle arrays," Phys. Rev. B 82, 1-12 (2010).

13. S. Jahani and Z. Jacob, "All-dielectric metamaterials," Nat. Nanotechnol. 11, 23-36 (2016).

14. M. A. Van De Haar, J. Van De Groep, B. J. M. Brenny, and A. Polman, "Controlling magnetic and electric dipole modes in hollow silicon nanocylinders," Opt. Express 24, 2047-2064 (2016).

15. D. Markovich, K. Baryshnikova, A. Shalin, A. Samusev, A. Krasnok, P. Belov, and P. Ginzburg, "Enhancement of artificial magnetism via resonant bianisotropy," Sci. Rep. 6, 22546 (2016).

16. A. B. Evlyukhin, C. Reinhardt, A. B. Evlyukhin, and B. N. Chichkov, "Multipole analysis of light scattering by arbitrary-shaped nanoparticles on a plane surface," J. Opt. Soc. Am. B 30, 2589-2598 (2013).

17. P. D. Terekhov, K. V. Baryshnikova, A. S. Shalin, A. Karabchevsky, and A. B. Evlyukhin, "Resonant forward scattering of light by highrefractive-index dielectric nanoparticles with toroidal dipole contribution," Opt. Lett. 42, 835-838 (2017).

18. D. R. Huffman and C. F. Bohren, Absorption and Scattering of Light by Small Particles (Wiley, 1998).
19. Q. Xu, V. R. Almeida, R. R. Panepucci, and M. Lipson, "Experimental demonstration of guiding and confining light in nanometer-size lowrefractive-index material," Opt. Lett. 29, 1626-1628 (2004).

20. M. I. Bakunov, A. V. Maslov, S. M. Kuznetsova, and S. N. Zhukov, "Magnetic response of planar dielectric rings," Photon. Nanostr. Fundam. Appl. 12, 114-121 (2014).

21. L. Jelinek and R. Marqués, "Artificial magnetism and left-handed media from dielectric rings and rods," J. Phys. Condens. Matter 22, 25902 (2010)

22. A. Andryieuski, S. M. Kuznetsova, and A. V. Lavrinenko, "Applicability of point-dipoles approximation to all-dielectric metamaterials," Phys. Rev. B 92, 035114 (2015).

23. A. B. Evlyukhin, S. M. Novikov, U. Zywietz, R. L. Eriksen, C. Reinhardt, S. I. Bozhevolnyi, and B. N. Chichkov, "Demonstration of magnetic dipole resonances of dielectric nanospheres in the visible region," Nano Lett. 12, 3749-3755 (2012).

24. A. B. Evlyukhin, T. Fischer, C. Reinhardt, and B. N. Chichkov, "Optical theorem and multipole scattering of light by arbitrary shaped nanoparticles," Phys. Rev. B 205434, 1-8 (2016).

25. D. E. Aspnes and A. A. Studna, "Dielectric functions and optical parameters of $\mathrm{Si}, \mathrm{Ge}, \mathrm{GaP}, \mathrm{GaAs}, \mathrm{GaSb}, \operatorname{InP}, \operatorname{InAs}$, and InSb from 1.5 to $6.0 \mathrm{eV}$," Phys. Rev. B 27, 985-1009 (1983).

26. A. B. Evlyukhin, C. Reinhardt, and B. N. Chichkov, "Multipole light scattering by nonspherical nanoparticles in the discrete dipole approximation," Phys. Rev. B 84, 1-8 (2011).

27. A. Mirzaei and A. E. Miroshnichenko, "Electric and magnetic hotspots in dielectric nanowire dimers," Nanoscale 7, 5963-5968 (2015).

28. Y. H. Fu, A. I. Kuznetsov, A. E. Miroshnichenko, Y. F. Yu, and B. Luk'yanchuk, "Directional visible light scattering by silicon nanoparticles," Nat. Commun. 4, 1527 (2013).

29. K. V. Baryshnikova, A. B. Evlyukhin, and A. S. Shalin, "Magnetic hotspots in hollow silicon cylinders," J. Phys. Conf. Ser. 741, 012156 (2016). 